\title{
HBED-NN: A Bifunctional Chelator for Constructing Radiopharmaceuticals
}

\author{
Ata Makarem*i \\ Karel D. Klika \\ German Litau ${ }^{\dagger}$ \\ Yvonne Remde ${ }^{\dagger}$ \\ Klaus Kopka ${ }^{\dagger, \S}$
}

${ }^{\dagger}$ German Cancer Research Center (DKFZ) Heidelberg, Division of Radiopharmaceutical Chemistry, INF 223, D69120 Heidelberg, Germany

†German Cancer Research Center (DKFZ) Heidelberg, Molecular Structure Analysis, INF 280, D-69120

Heidelberg, Germany

${ }^{\S}$ German Cancer Consortium (DKTK), Heidelberg, Germany

*E-mail: a.makarem@dkfz.de

\section{Table of Contents}

1. Click Chemistry of HBED-NN (1) ............................................................................ SI-2

2. Complex Geometry Determination of [Ga-(HBED-NN)] (3) ............................................ SI-3

3. NMR Spectra $\left({ }^{1} \mathrm{H},{ }^{13} \mathrm{C}\right.$, COSY, HSQC, HMBC, NOESY) .................................................. SI-7 


\section{Click Chemistry of HBED-NN (1)}

Table S1. CuAAC Reactions of HBED-NN (1)
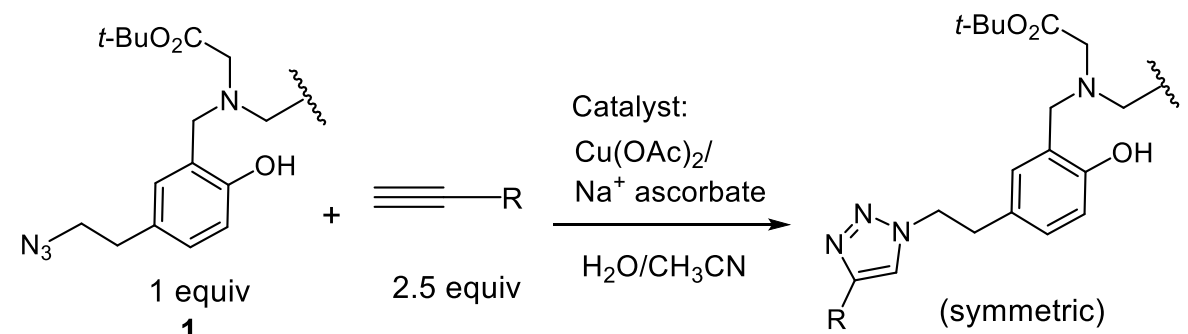

1aa : $\mathrm{R}=\mathrm{Ph}, 1 \mathbf{a b}: \mathrm{R}=\mathrm{CO}_{2} \mathrm{Et}, 1 \mathrm{ac}: \mathrm{R}=\mathrm{CH}_{2} \mathrm{OH}$

\begin{tabular}{|c|c|c|c|c|c|}
\hline $\mathrm{R}$ & $\begin{array}{c}\mathrm{Cu}(\mathrm{OAc})_{2} \\
\text { (equiv) }\end{array}$ & $\begin{array}{c}\mathrm{Na}^{+} \\
\text {ascorbate } \\
\text { (equiv) }\end{array}$ & $\begin{array}{c}t^{a} \\
(\mathrm{~min})\end{array}$ & $\begin{array}{c}T \\
\left({ }^{\circ} \mathrm{C}\right)\end{array}$ & $\begin{array}{c}\text { Yield }^{b} \\
(\%)\end{array}$ \\
\hline $\mathrm{Ph}$ & 0.05 & 0.10 & 15 & 80 & 59 \\
\hline $\mathrm{CO}_{2} \mathrm{Et}$ & 0.05 & 0.10 & 10 & 80 & 43 \\
\hline $\mathrm{CH}_{2} \mathrm{OH}$ & 0.05 & 0.10 & 30 & 80 & 50 \\
\hline $\mathrm{Ph}$ & 0.5 & 1.0 & 15 & r.t. & 47 \\
\hline $\mathrm{Ph}$ & 0.05 & 0.10 & $\begin{array}{c}>2 \\
\text { days }\end{array}$ & r.t. & - \\
\hline $\mathrm{Ph}$ & $0.05^{c}$ & 0.10 & $\begin{array}{c}>2 \\
\text { days }\end{array}$ & r.t. & - \\
\hline
\end{tabular}

${ }^{a}$ Completion of the reaction was determined by HPLC or TLC.

${ }^{b}$ Isolated yield.

${ }^{c}$ Reaction conducted with 0.10 equiv HOAc. 


\section{Complex Geometry Determination of [Ga-(HBED-NN)] (3)}

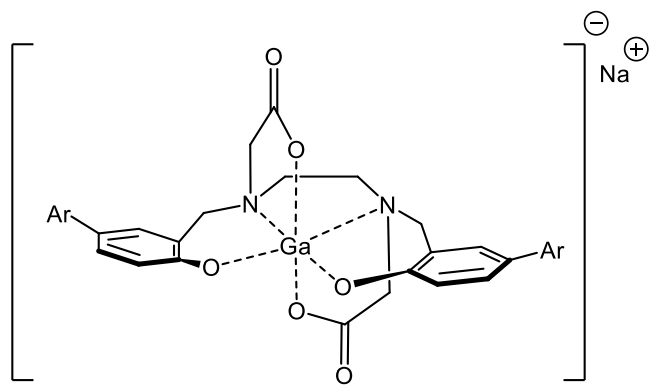

isomer II (favored structure)

Table S2. The HMBC correlations for the three-bond couplings observed for complex $\mathbf{3}$ involving the protons on the $\alpha$ carbons to the coordinating nitrogen atom and the other two $\alpha$ carbons in relation to their expected appearance based on the dihedral angle between the nuclei concerned

\begin{tabular}{|c|c|c|c|c|c|}
\hline group & $\begin{array}{l}\text { proton } \\
(\mathrm{ppm})\end{array}$ & $\begin{array}{l}\text { carbon } \\
(\mathrm{ppm})\end{array}$ & $\begin{array}{l}\text { dihedral } \\
\text { angle }\end{array}$ & $\begin{array}{c}\text { expectation } \\
\text { (yes, to be present/ } \\
\text { no, not to be present) }\end{array}$ & $\begin{array}{l}\text { result } \\
\text { (observed } / \\
\text { not observed } \mathbf{X} \text { ) } \\
\end{array}$ \\
\hline \multirow[t]{4}{*}{ benzylic } & $1(4.2)$ & carboxylic & high & yes & \\
\hline & $2(3.5)$ & (55.9) & medium & no & $\gamma$ \\
\hline & $1(4.2)$ & bridge & medium & no & \\
\hline & $2(3.5)$ & (55.3) & medium & no & \\
\hline \multirow[t]{4}{*}{ carboxylic } & $1(2.9)$ & benzylic & medium & no & $\mathbf{X}$ \\
\hline & $2(3.2)$ & $(60.8)$ & low & yes & \\
\hline & $1(2.9)$ & bridge & low & yes & \\
\hline & $2(3.2)$ & (55.3) & high & yes & \\
\hline \multirow[t]{4}{*}{ bridge } & $1(2.8)$ & carboxylic & high & yes & \\
\hline & $2(2.8)$ & (55.9) & low & yes & \\
\hline & $1(2.8)$ & benzylic & medium & no & 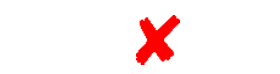 \\
\hline & $2(2.8)$ & $(60.8)$ & medium & no & Y \\
\hline
\end{tabular}




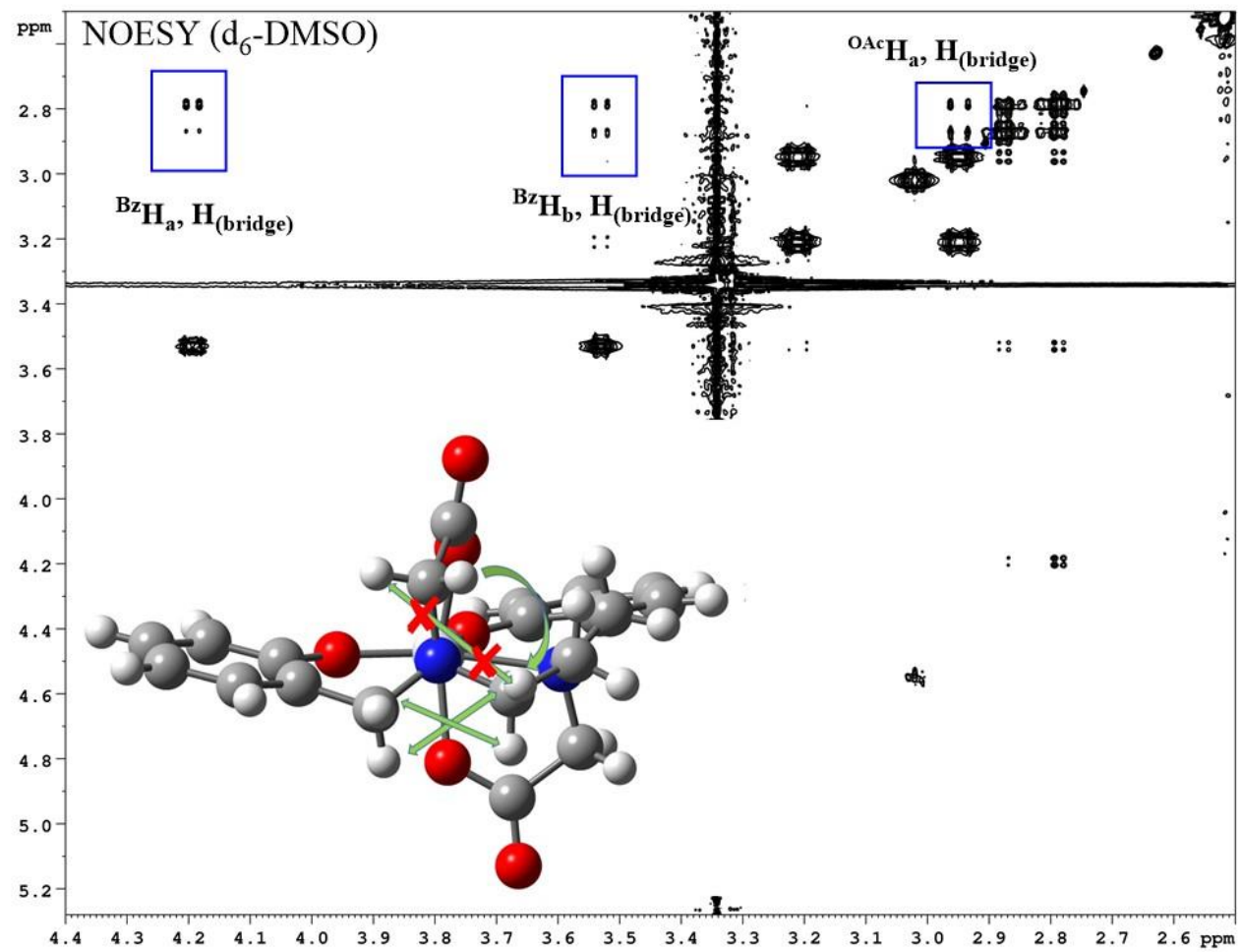

Figure S1. Example portion of the NOESY spectrum for complex 3 (isomer II). Only one proton of the methylene adjacent to the carboxylate group has sizeable NOE's with the protons of the $-\mathrm{NCH}_{2} \mathrm{CH}_{2} \mathrm{~N}$ - bridge, while both protons of the benzylic methylene have sizeable NOE's with the protons of the $-\mathrm{NCH}_{2} \mathrm{CH}_{2} \mathrm{~N}-$ bridge. Colors: $\mathrm{C}$ gray, $\mathrm{O}$ red, $\mathrm{N}$ blue, $\mathrm{H}$ light gray. 


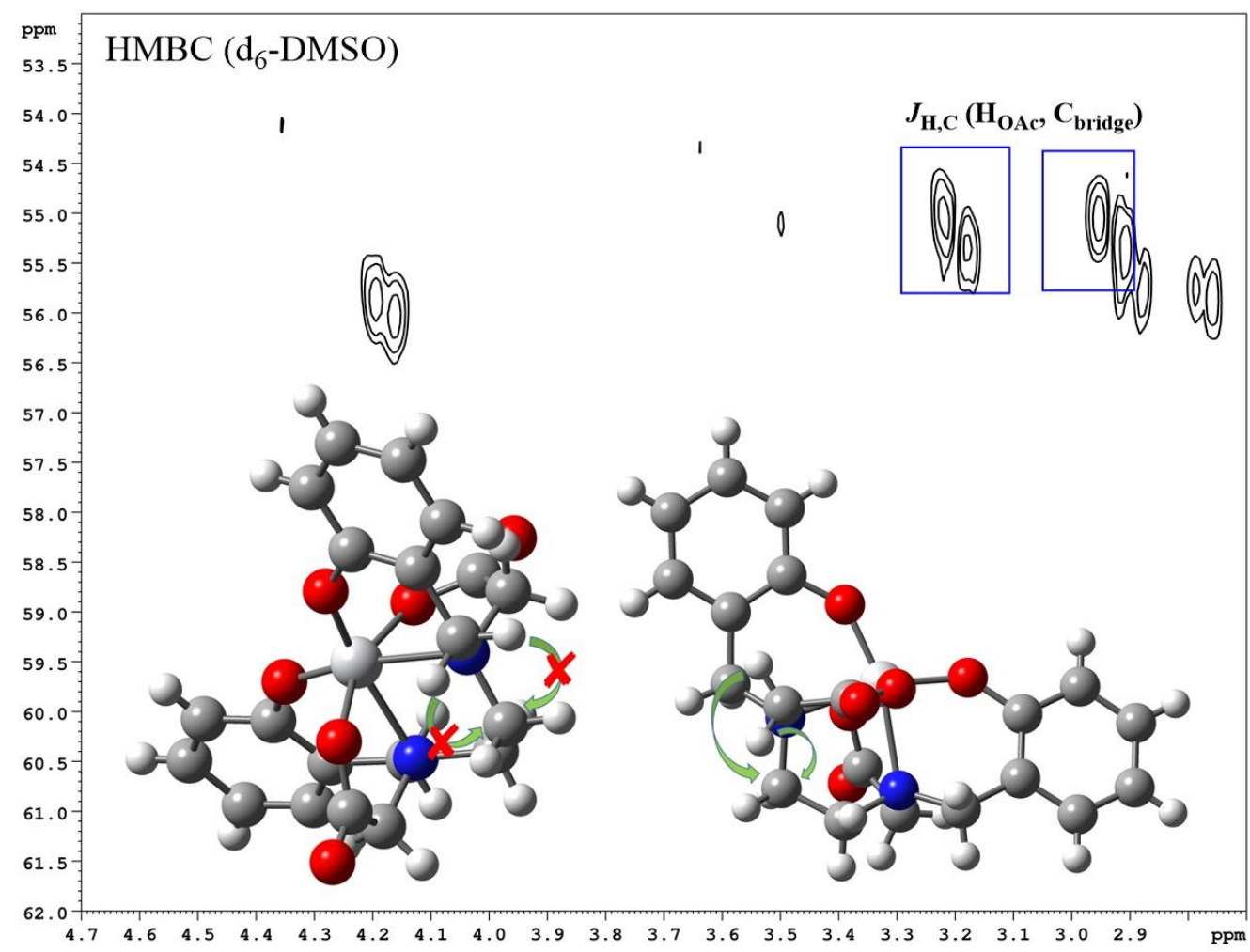

Figure S2. Example portion of the HMBC spectrum for complex 3 (isomer II). The two methylene protons adjacent to the carboxylate group provided correlations to the $-\mathrm{NCH}_{2} \mathrm{CH}_{2} \mathrm{~N}$ - bridge carbons (right). Neither of the benzylic methylene protons makes such correlations (left), since they are not suitably disposed in terms of dihedral angles. Colors: $\mathrm{C}$ gray, $\mathrm{O}$ red, $\mathrm{N}$ blue, $\mathrm{H}$ light gray. 

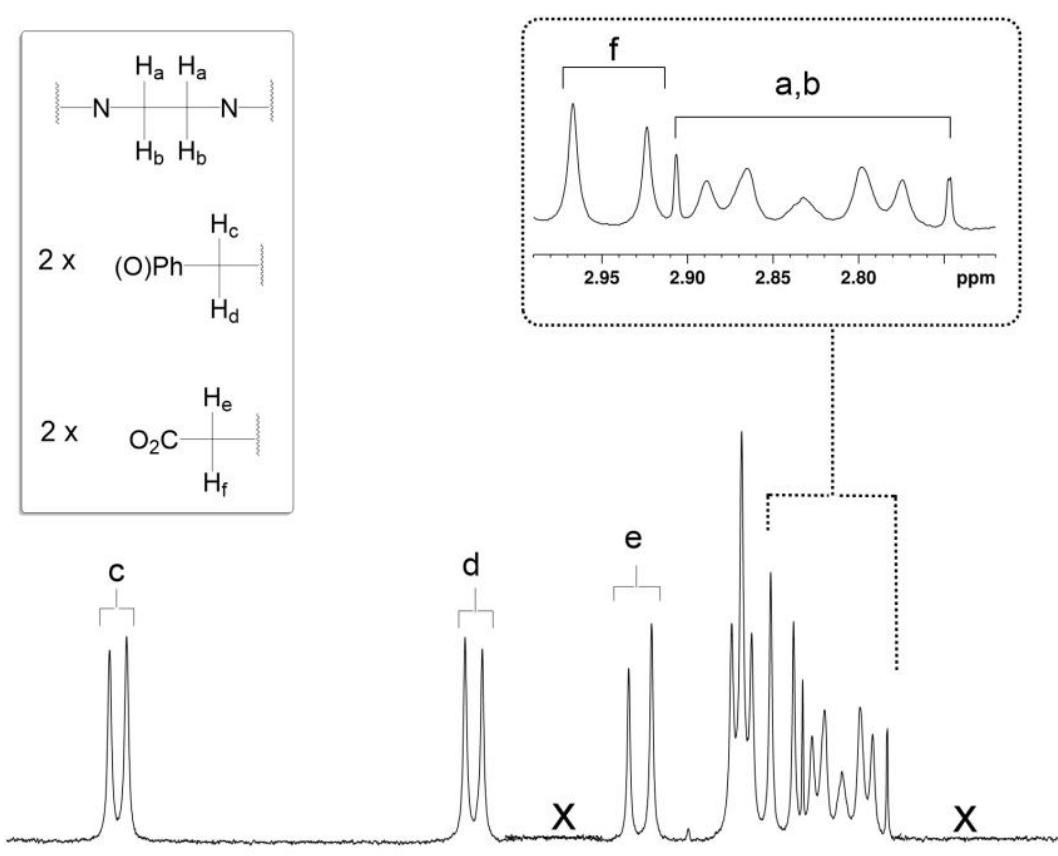

$\begin{array}{lllllllllllllllllllll}4 & 4.3 & 4.2 & 4.1 & 4.0 & 3.9 & 3.8 & 3.7 & 3.6 & 3.5 & 3.4 & 3.3 & 3.2 & 3.1 & 3.0 & 2.9 & 2.8 & 2.7 & 2.6 & 2.5 & 2\end{array}$

Figure S3. ${ }^{1} \mathrm{H}$ NMR ( $\left.400 \mathrm{MHz}, \mathrm{d}_{6}-\mathrm{DMSO}\right)$ spectrum for diastereotopic protons of the chelate cage of complex $\mathbf{3}$. For more clarity solvent peaks $(\mathbf{x})$ were removed.

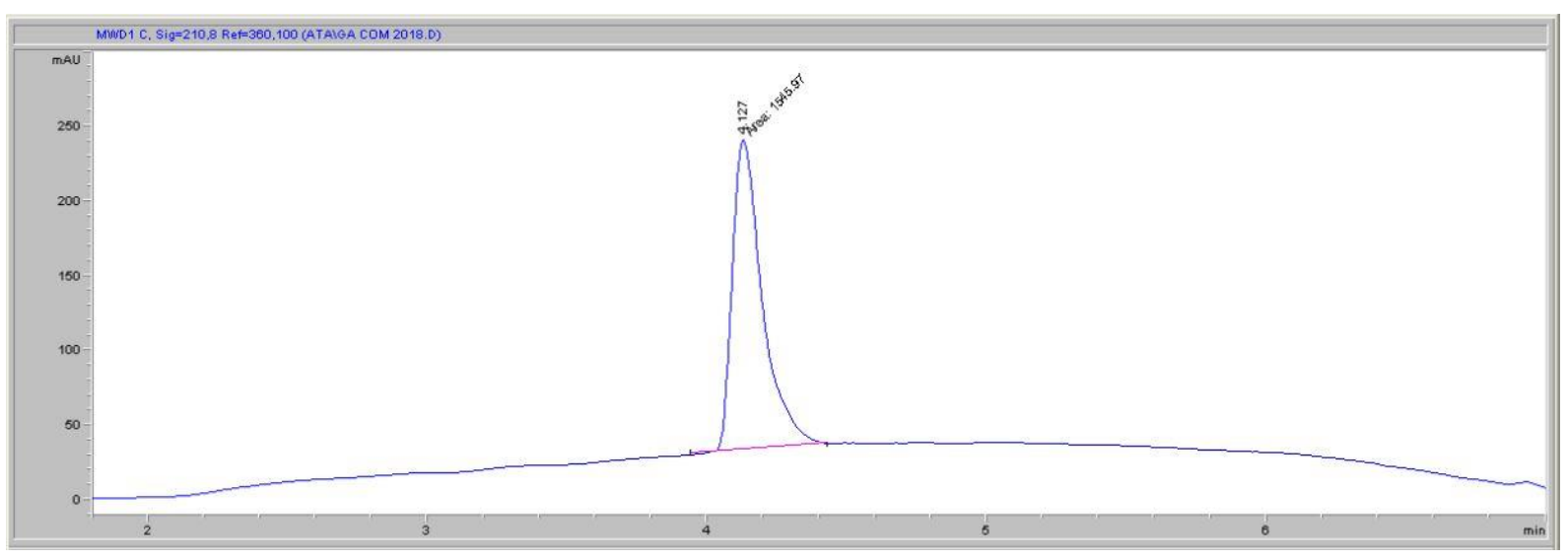

Figure S4. HPLC chromatogram of complex 3 (isomer II). 
3. NMR Spectra $\left({ }^{1} \mathrm{H},{ }^{13} \mathrm{C}, \mathrm{COSY}, \mathrm{HSQC}, \mathrm{HMBC}, \mathrm{NOESY}\right)$

${ }^{1} \mathrm{H}$ NMR $\left(\mathrm{CDCl}_{3}\right)$
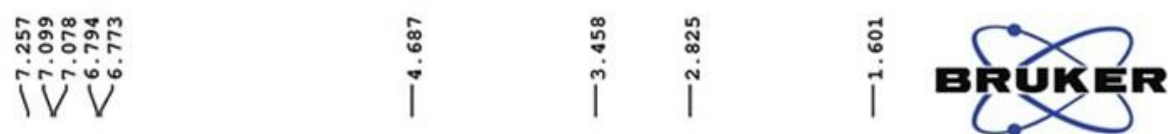<smiles>N#CCCc1ccc(O)cc1</smiles>

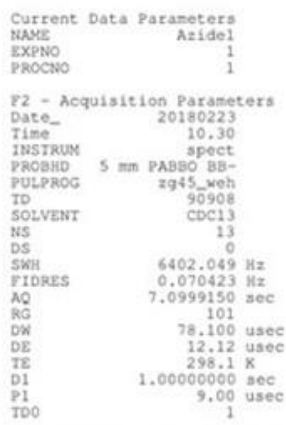

1b

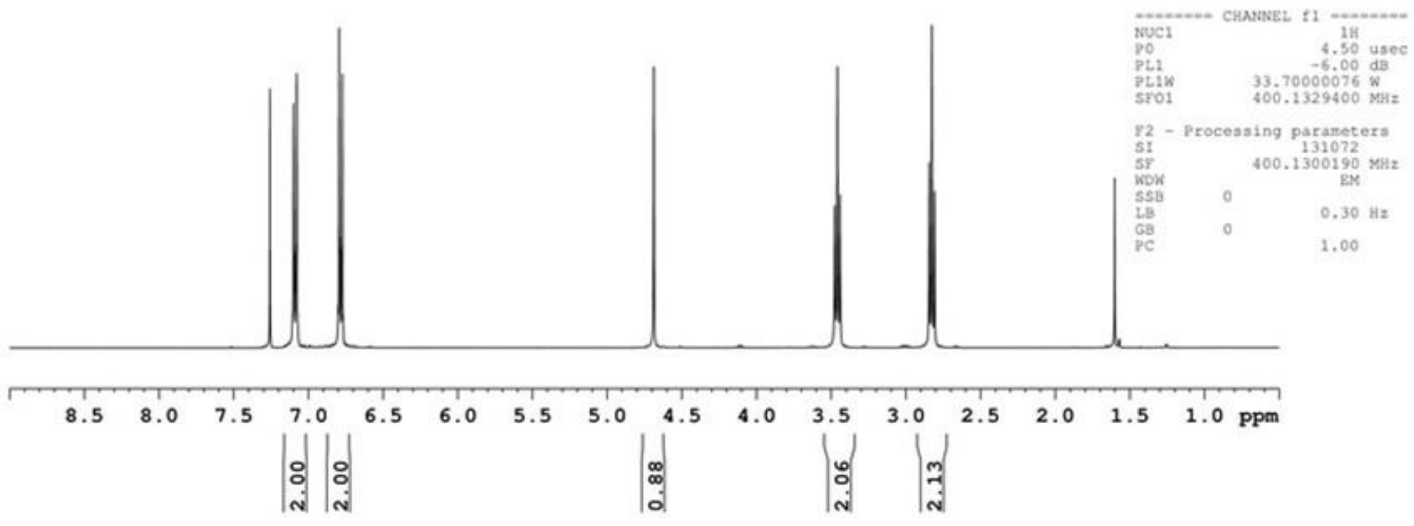

${ }^{13} \mathrm{C} \mathrm{NMR}\left(\mathrm{CDCl}_{3}\right)$

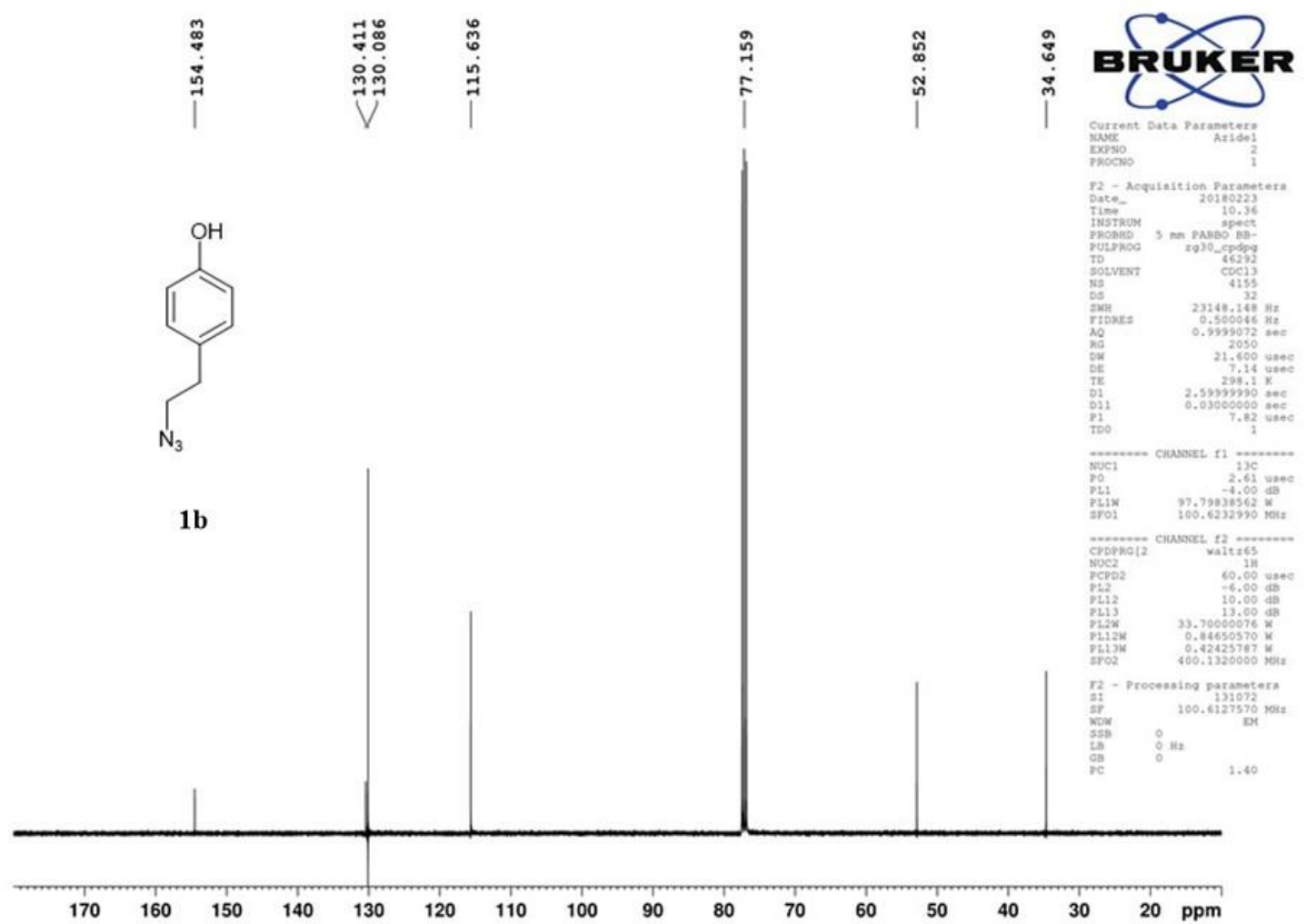


${ }^{1} \mathrm{H}$ NMR $\left(\mathrm{CDCl}_{3}\right)$

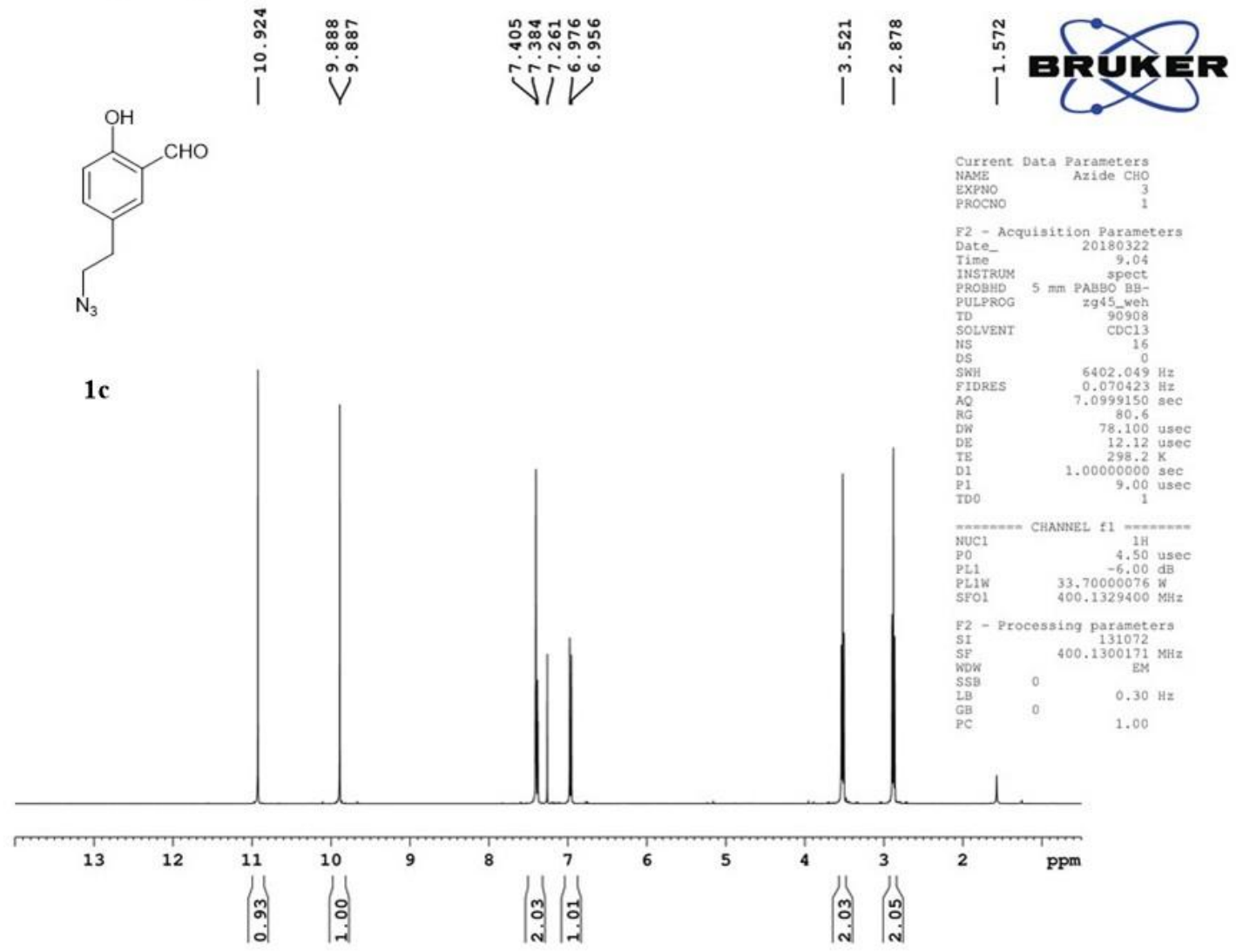

${ }^{13} \mathrm{C} \mathrm{NMR}\left(\mathrm{CDCl}_{3}\right)$

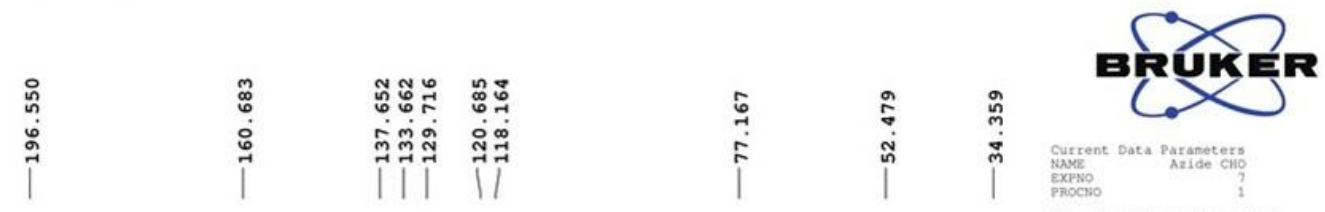<smiles>N#CCc1ccc(O)c(C=O)c1</smiles>

$1 \mathrm{c}$

$\begin{array}{llllllllllllllllllll}210 & 200 & 190 & 180 & 170 & 160 & 150 & 140 & 130 & 120 & 110 & 100 & 90 & 80 & 70 & 60 & 50 & 40 & 30 & \mathrm{ppm}\end{array}$ 
${ }^{1} \mathrm{H} \operatorname{NMR}\left(\mathrm{CDCl}_{3}\right)$

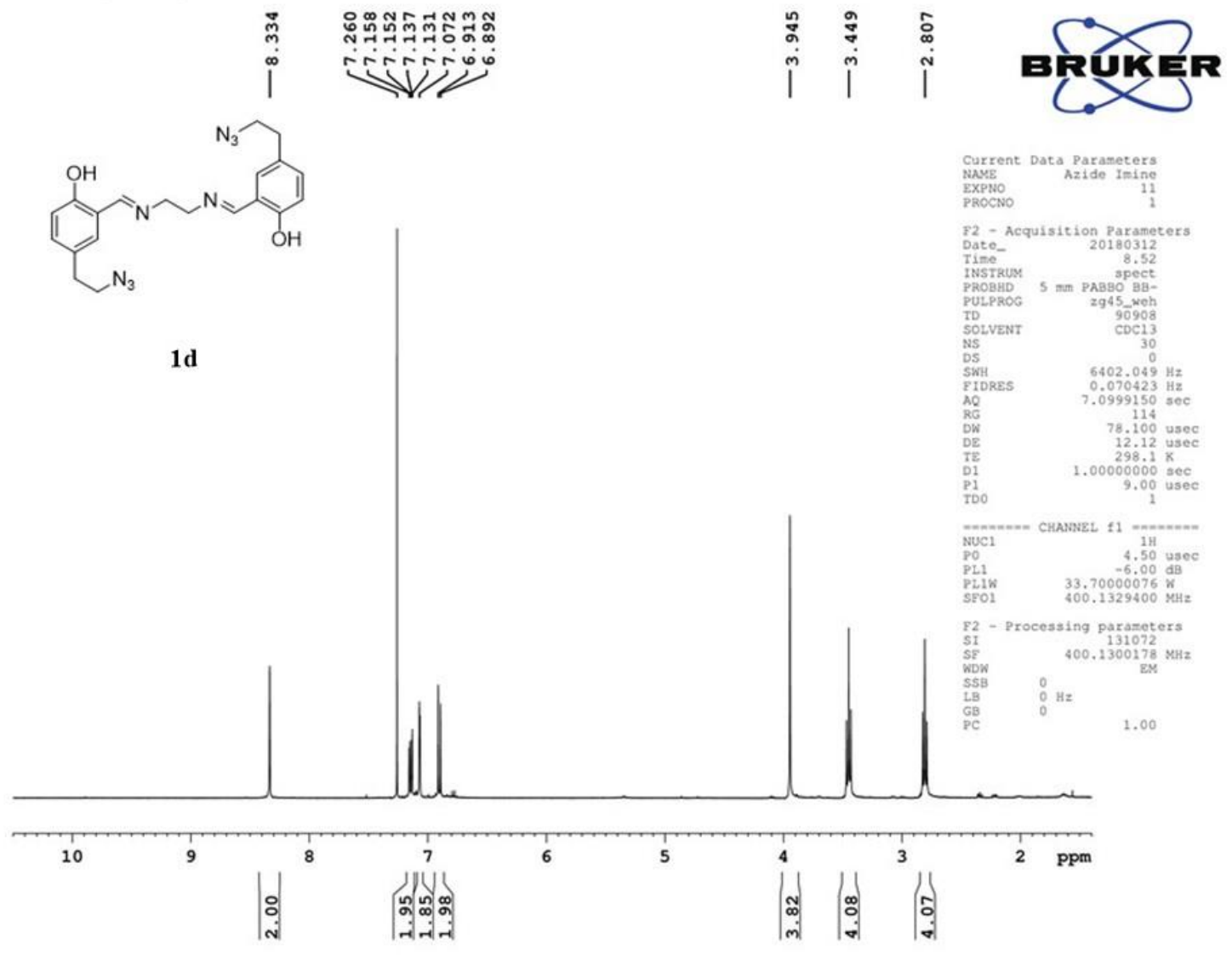

${ }^{13} \mathrm{C} \mathrm{NMR}\left(\mathrm{CDCl}_{3}\right)$

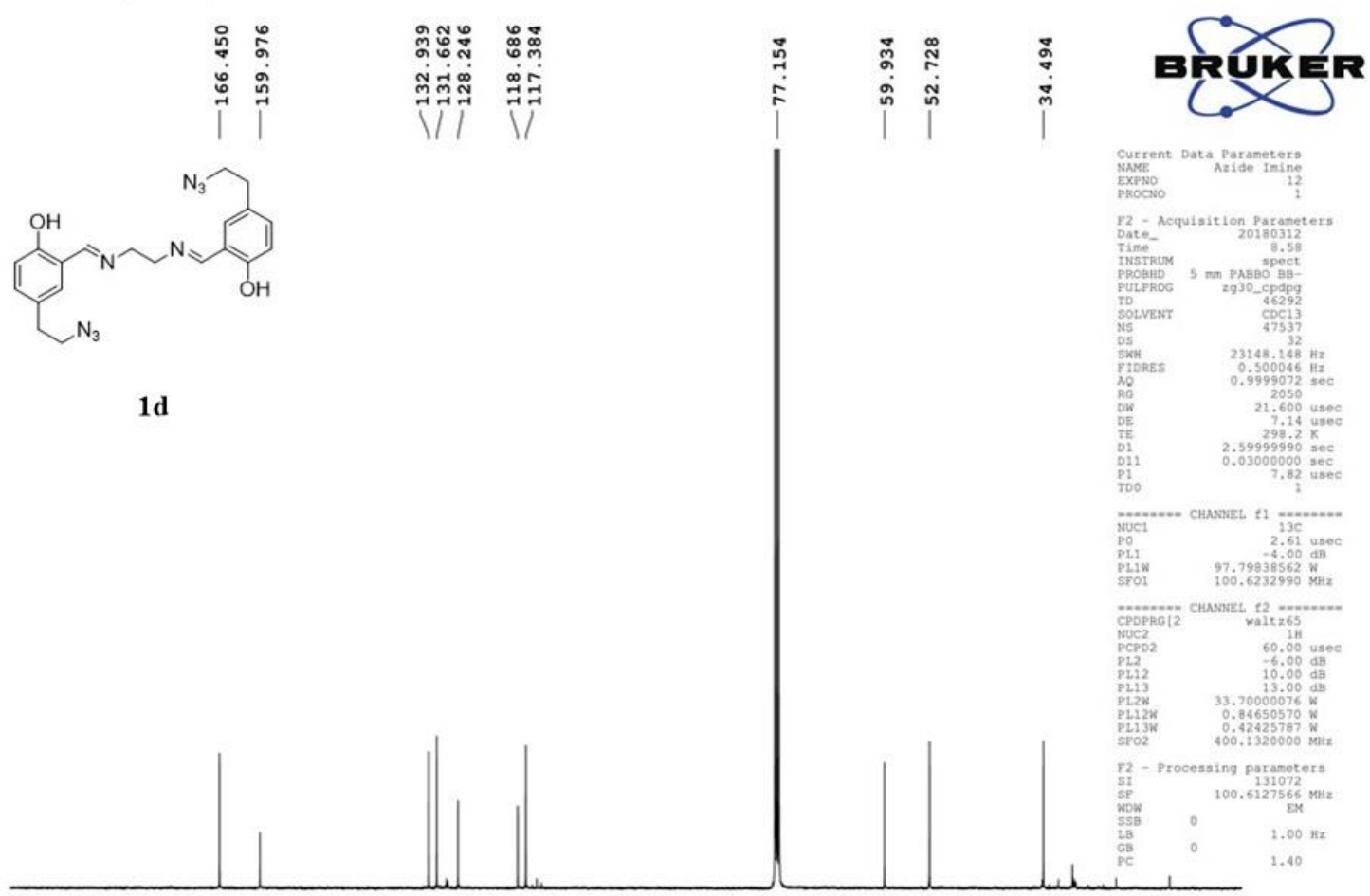

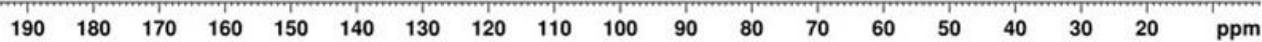


${ }^{1} \mathrm{H} \mathrm{NMR}\left(\mathrm{CDCl}_{3}\right)$

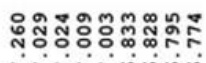

다유유

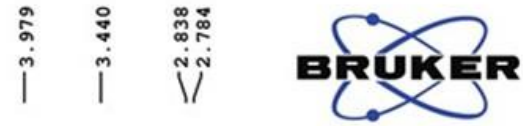

$\mathrm{C}_{\mathrm{N}_{3}}^{(}$

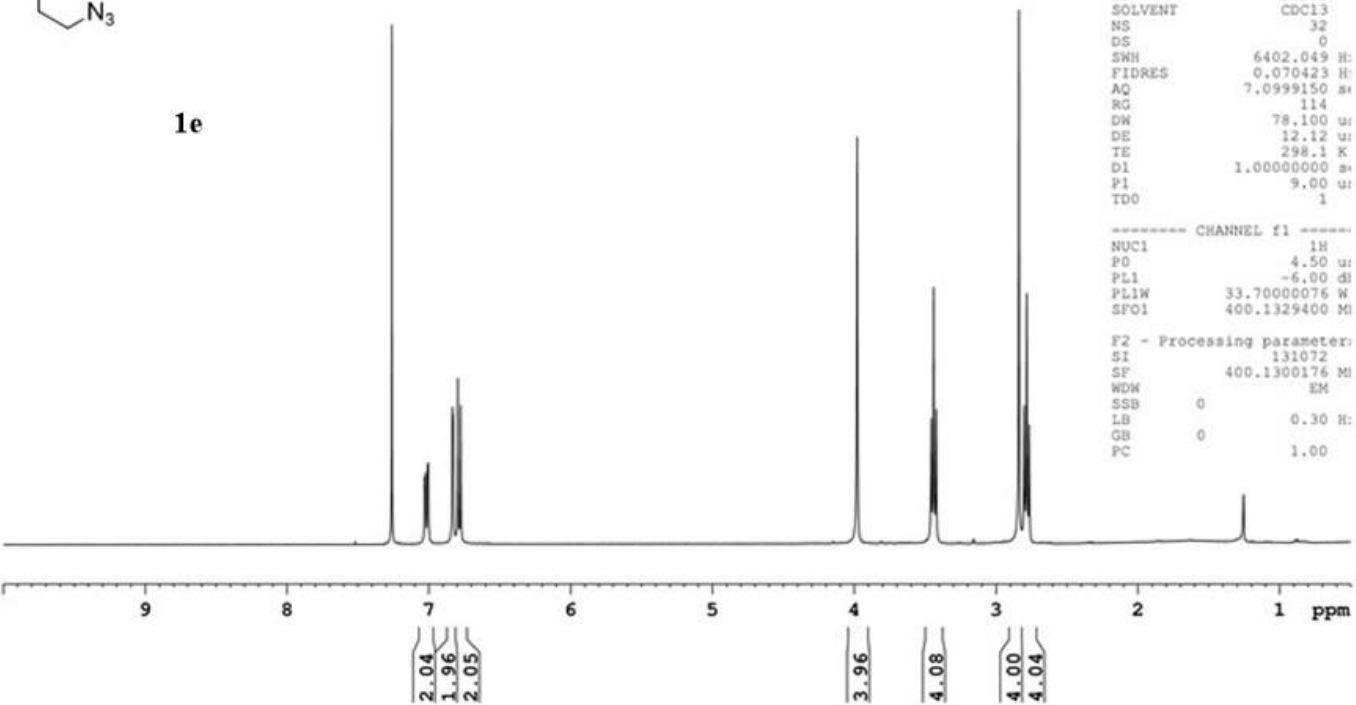

${ }^{13} \mathrm{C} \mathrm{NMR}\left(\mathrm{CDCl}_{3}\right)$

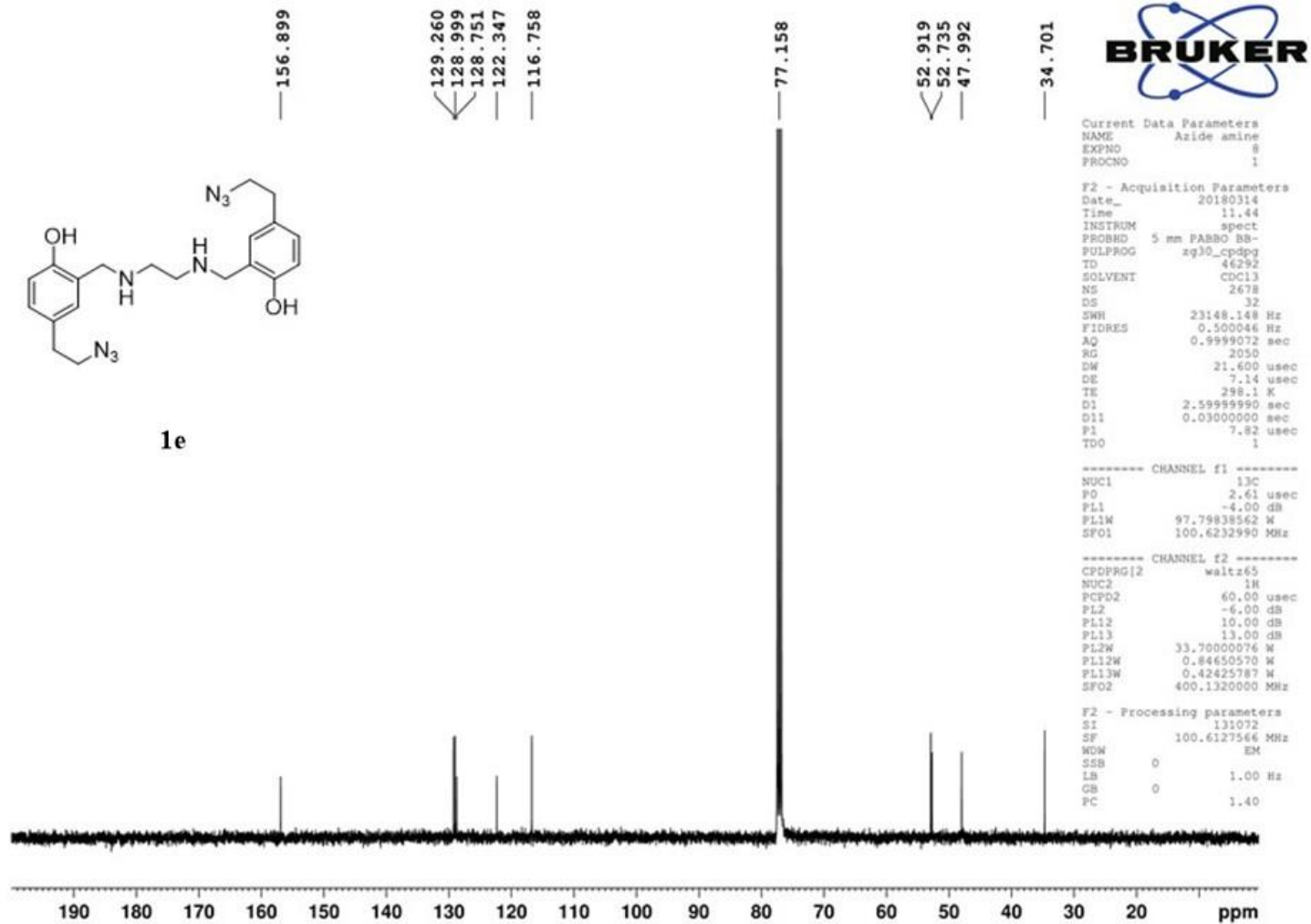


${ }^{1} \mathrm{H} \mathrm{NMR}\left(\mathrm{CDCl}_{3}\right)$

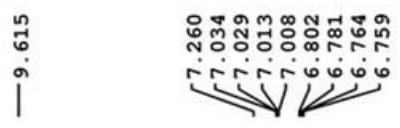<smiles>CC(C)(C)OC(=O)CN(CCN(CC(=O)OCc1ccccc1)Cc1cc(CC#N)ccc1O)Cc1cc(CCN)ccc1O</smiles>

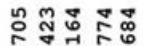
ขंगขं

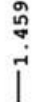

BRUKER

rrent Data Parameters

NAME Azide di (t-Bu ester

EXPNO

F2 - Acquisition Parameters
Date_ 20180315

Time

INSTRUM
PROBHD $5 \mathrm{~mm}$ PABBO BB-

PROBHD 5 mm PABBO BB-
PULPROG
zg45 weh

SOLVENT

$\begin{array}{ll}\text { DS } & 0 \\ \text { SWH } & 6402.049 \mathrm{~Hz} \\ \text { FIDRES } & 0.070423 \mathrm{~Hz}\end{array}$

$\begin{array}{ll}0.070423 \mathrm{~Hz} \\ \text { AQ } & 7.0999150 \mathrm{sec}\end{array}$

RG

$7.0999150 \mathrm{sec}$
71.8

78.100 usec
12.12 usec

$\begin{array}{lr}\mathrm{TE} & 298.1 \mathrm{~K} \\ \mathrm{~T} 1 & 1.00000000 \mathrm{sec} \\ \mathrm{P} 1 & 9.00 \mathrm{usec}\end{array}$

TDO

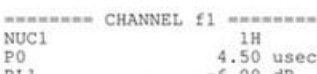

di

SEO1 $\quad \begin{aligned} & 33.70000076 \\ & 400.1329400 \mathrm{MHz}\end{aligned}$

F2 - Processing parameters

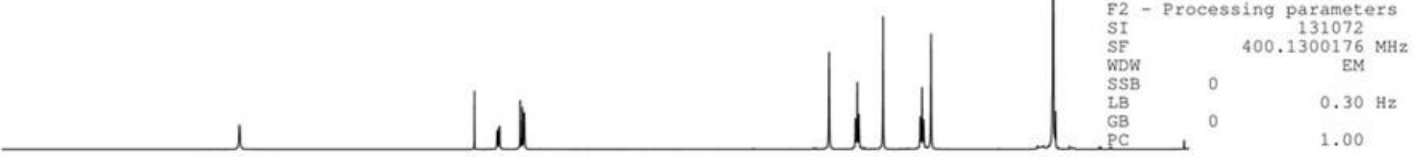

11
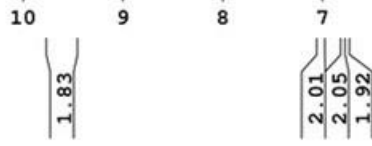

ข่ำ่ำ

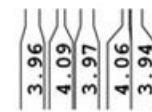

:

${ }^{13} \mathrm{C} \mathrm{NMR}\left(\mathrm{CDCl}_{3}\right)$

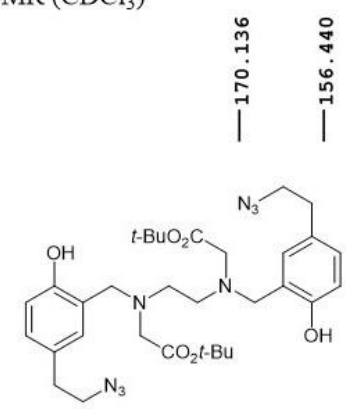

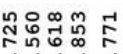

สํㅝำ

Vर । ।

\%

i i

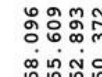

ผู่ ถู่

กั

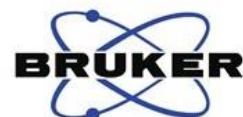

। ।

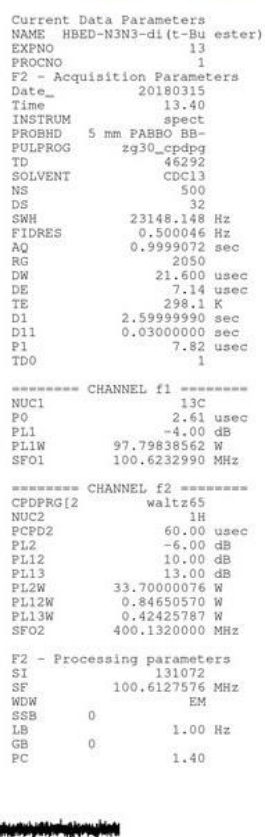

$\begin{array}{lllllllllllllllllllll}210 & 200 & 190 & 180 & 170 & 160 & 150 & 140 & 130 & 120 & 110 & 100 & 90 & 80 & 70 & 60 & 50 & 40 & 30 & 20 & \mathrm{ppm}\end{array}$ 
${ }^{1} \mathrm{H} \mathrm{NMR}\left(\mathrm{CDCl}_{3}\right)$
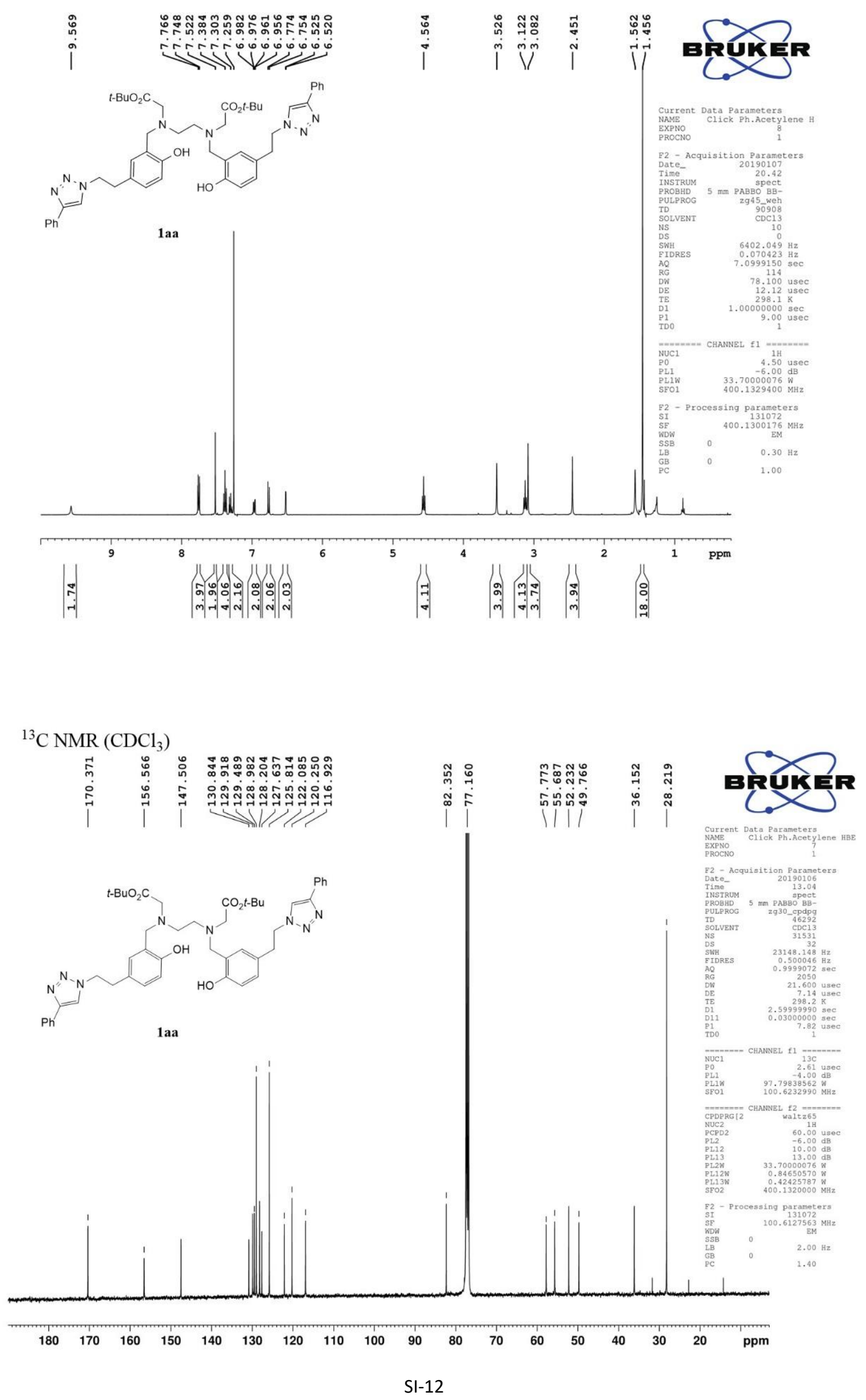

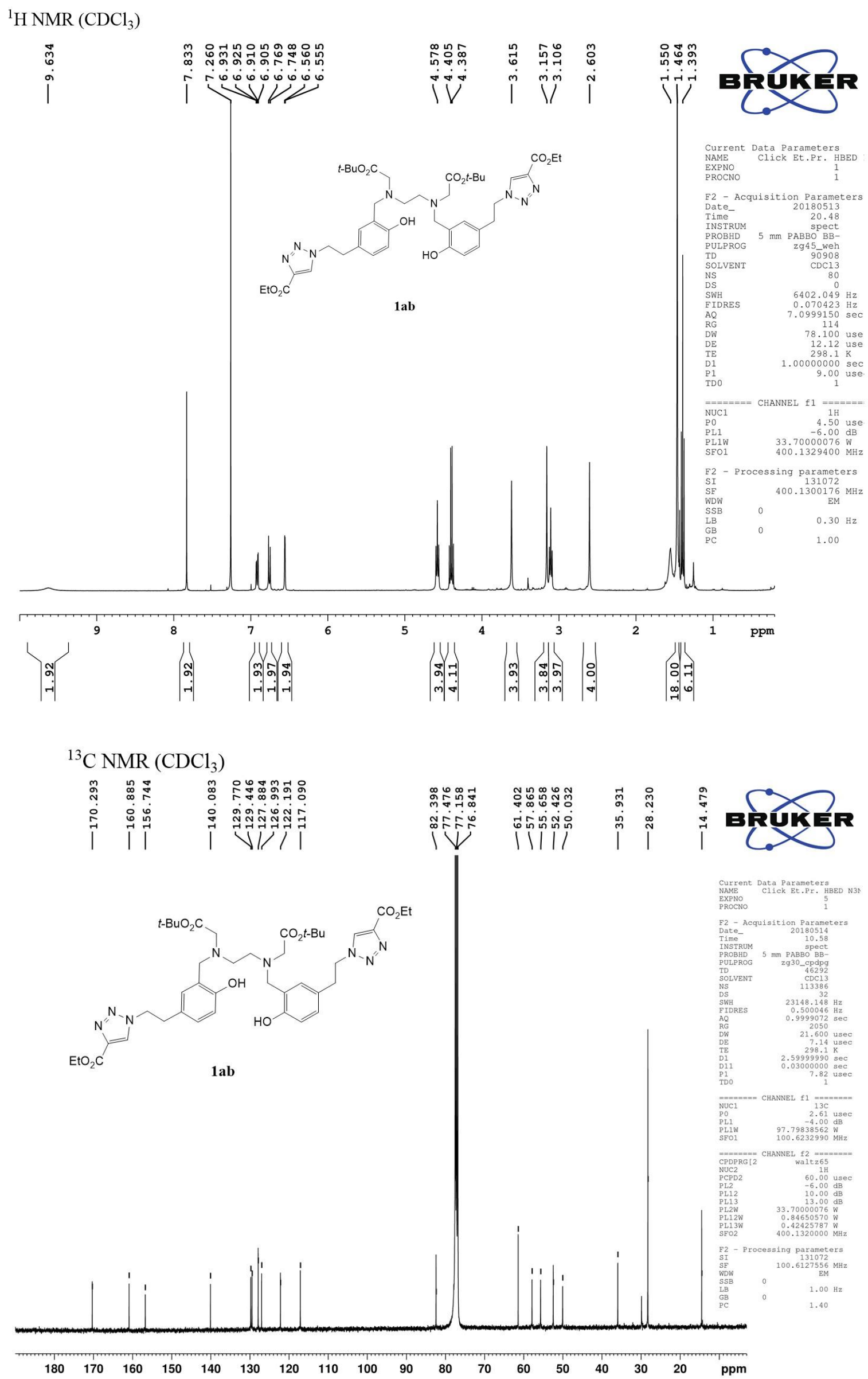


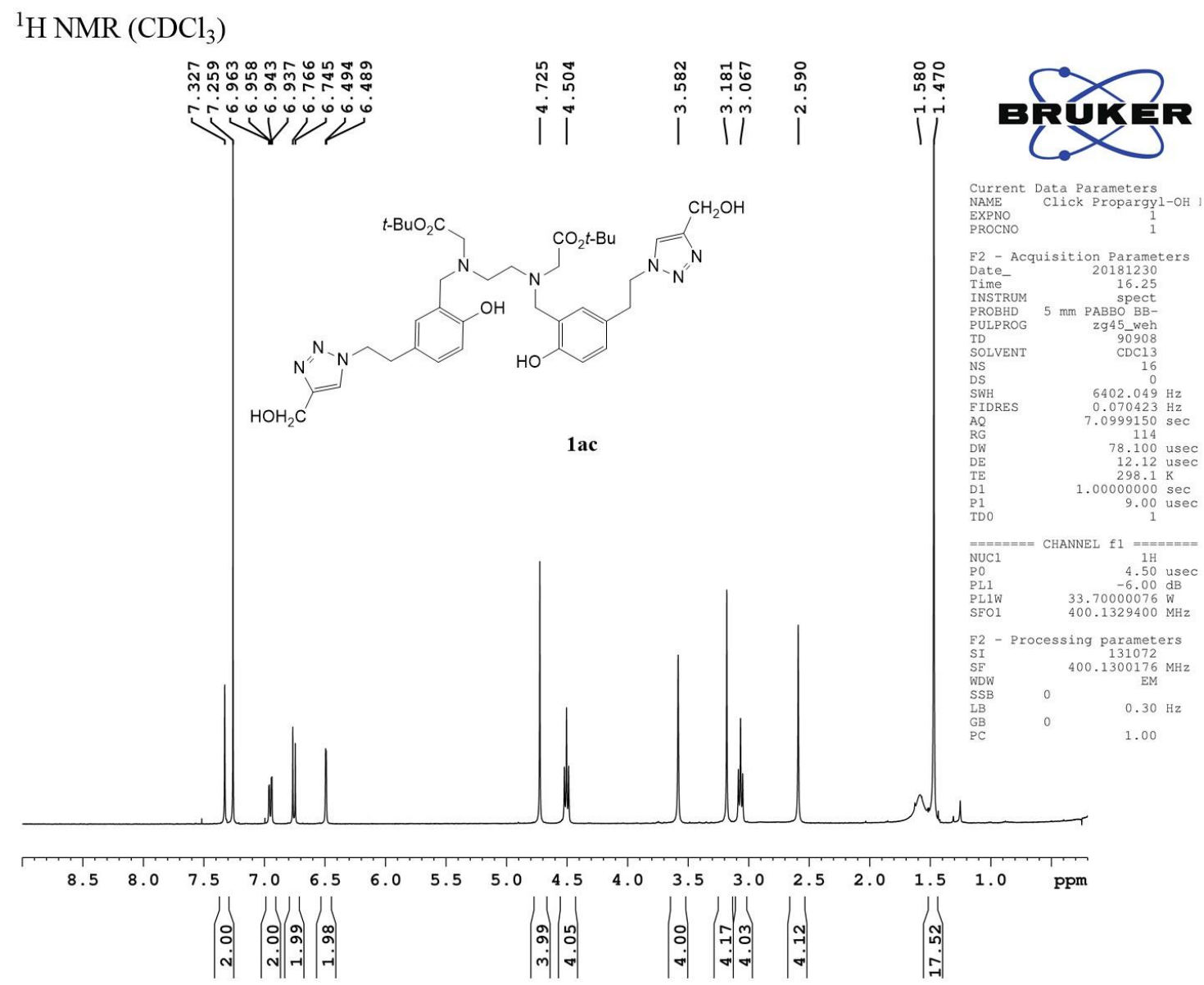

${ }^{13} \mathrm{C} \mathrm{NMR}\left(\mathrm{CDCl}_{3}\right)$

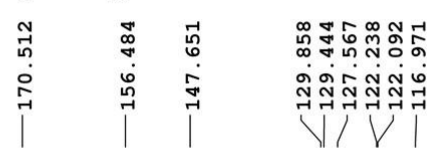

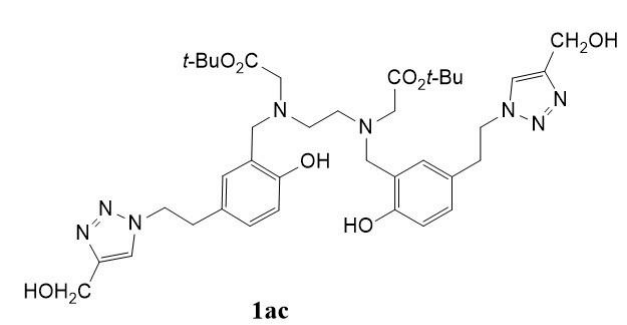

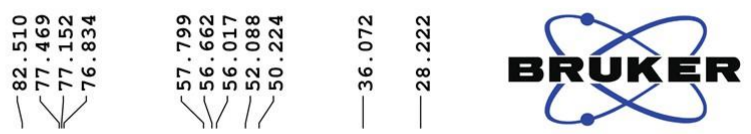

Current Data Parameters
NAME Click Propargy1-OH HBE
EXPNO

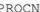

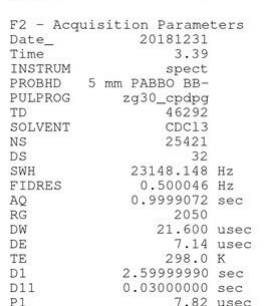

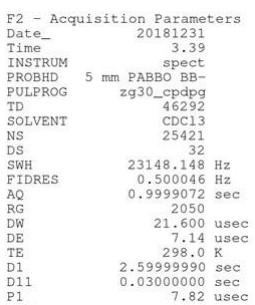

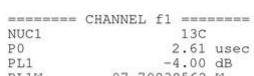

$-4.00 \mathrm{~dB}$
PL1W
SEO1

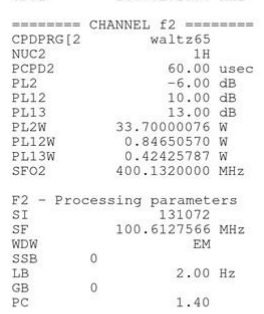

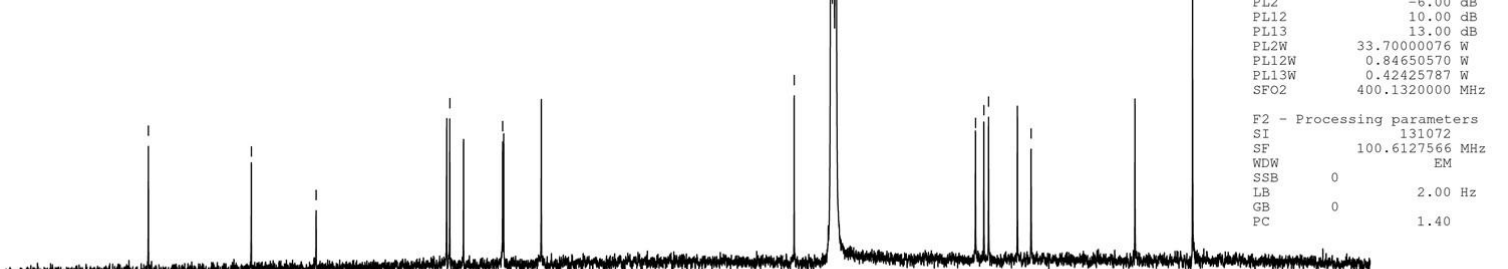

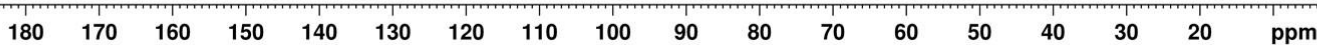




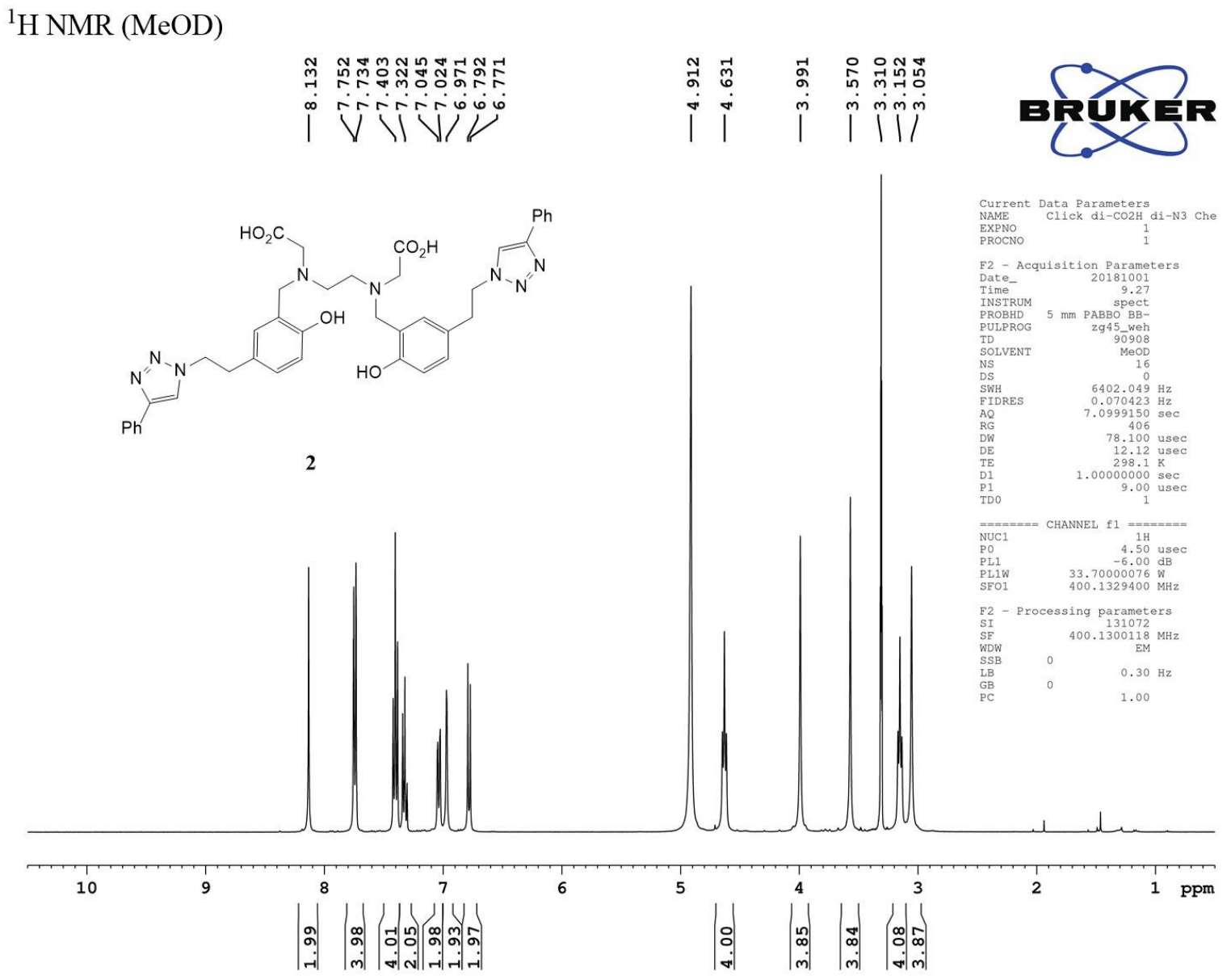

${ }^{13} \mathrm{C}$ NMR (MeOD)
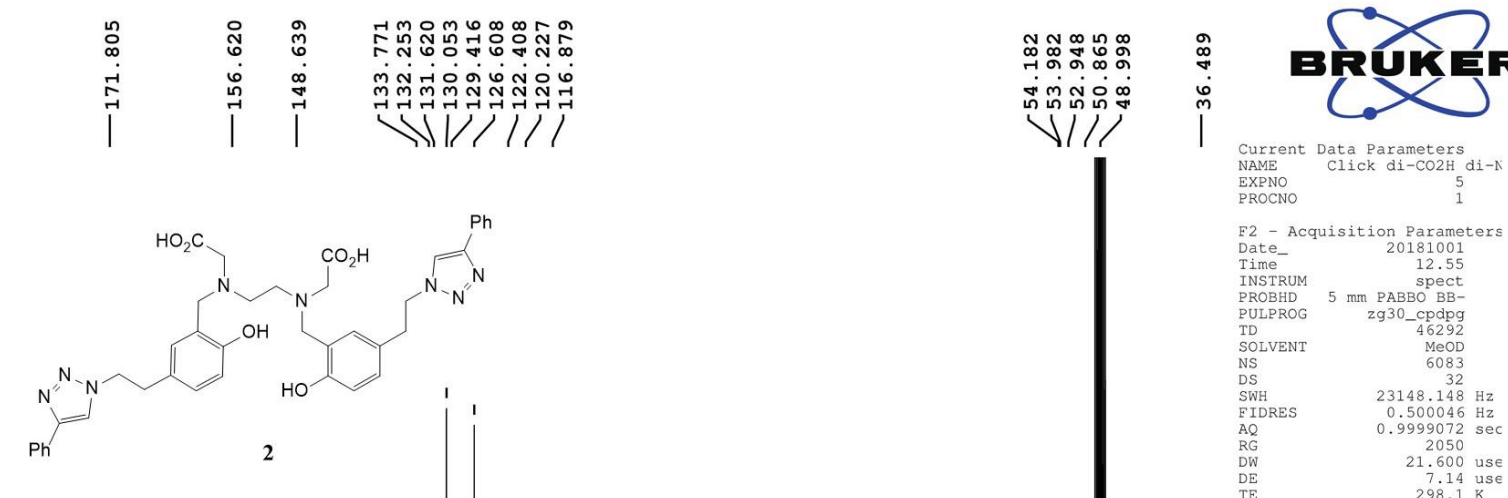
ถู่ ํำกำ

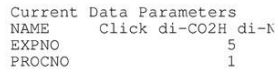

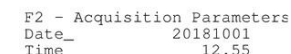

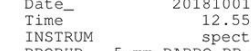

PROBHD $5 \mathrm{~mm}$ PABBO BB-
PUIPROG
Zq30 Cpdo

SOLVENT

NS
DS
SWH

$\begin{array}{ll}\text { SWH } & 32 \\ \text { EIDRES } & 23148.148 \mathrm{HZ} \\ \text { IDRE } & 0.5500645\end{array}$

AO

DE

$\mathrm{T}$
$\mathrm{T}$
$\mathrm{D} 11$
$\mathrm{D} 11$
$\mathrm{P}$
$\mathrm{TDO}$

$0.9999072 \mathrm{sec}$
2050

7.14 use
$298.10 \mathrm{~K}$
2.58000

$2.59999990 \mathrm{sec}$
$0.0300000 \mathrm{sec}$

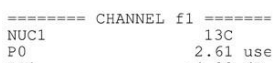

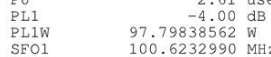

CPDPRG [2

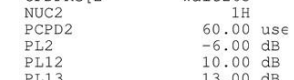

P P P

PLI2W
PLI

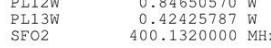

F2 - Processing parameters $100.6126291 \mathrm{MHz}$

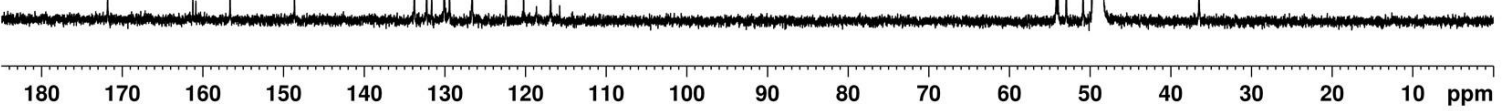


${ }^{1} \mathrm{H}$ NMR $\left(\mathrm{d}_{6}-\mathrm{DMSO}\right)$

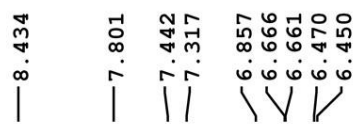

$\mathrm{SP}=$ solvent peaks
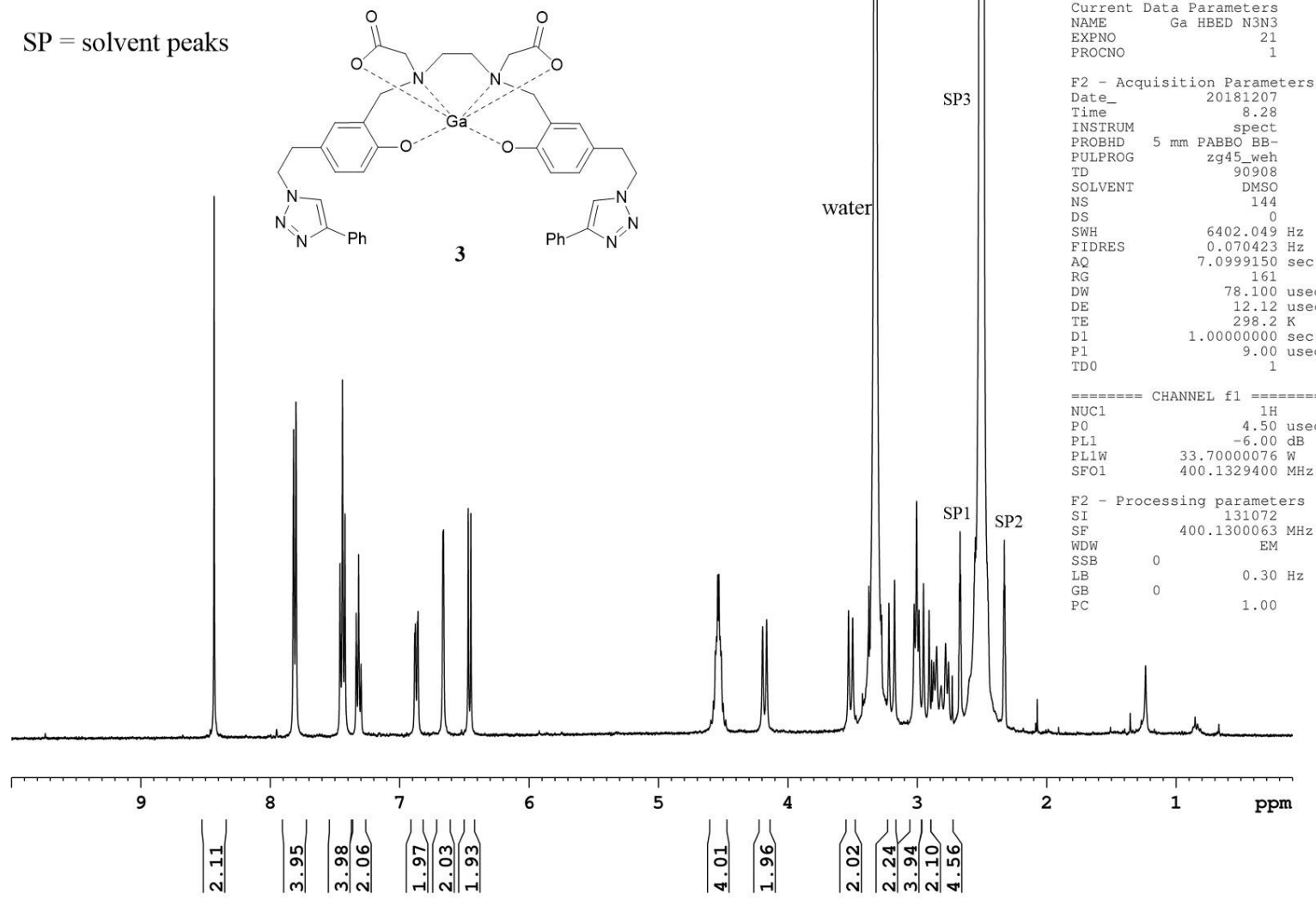

${ }^{13} \mathrm{C} \mathrm{NMR}\left(\mathrm{d}_{6}\right.$-DMSO)

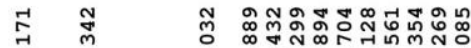

|
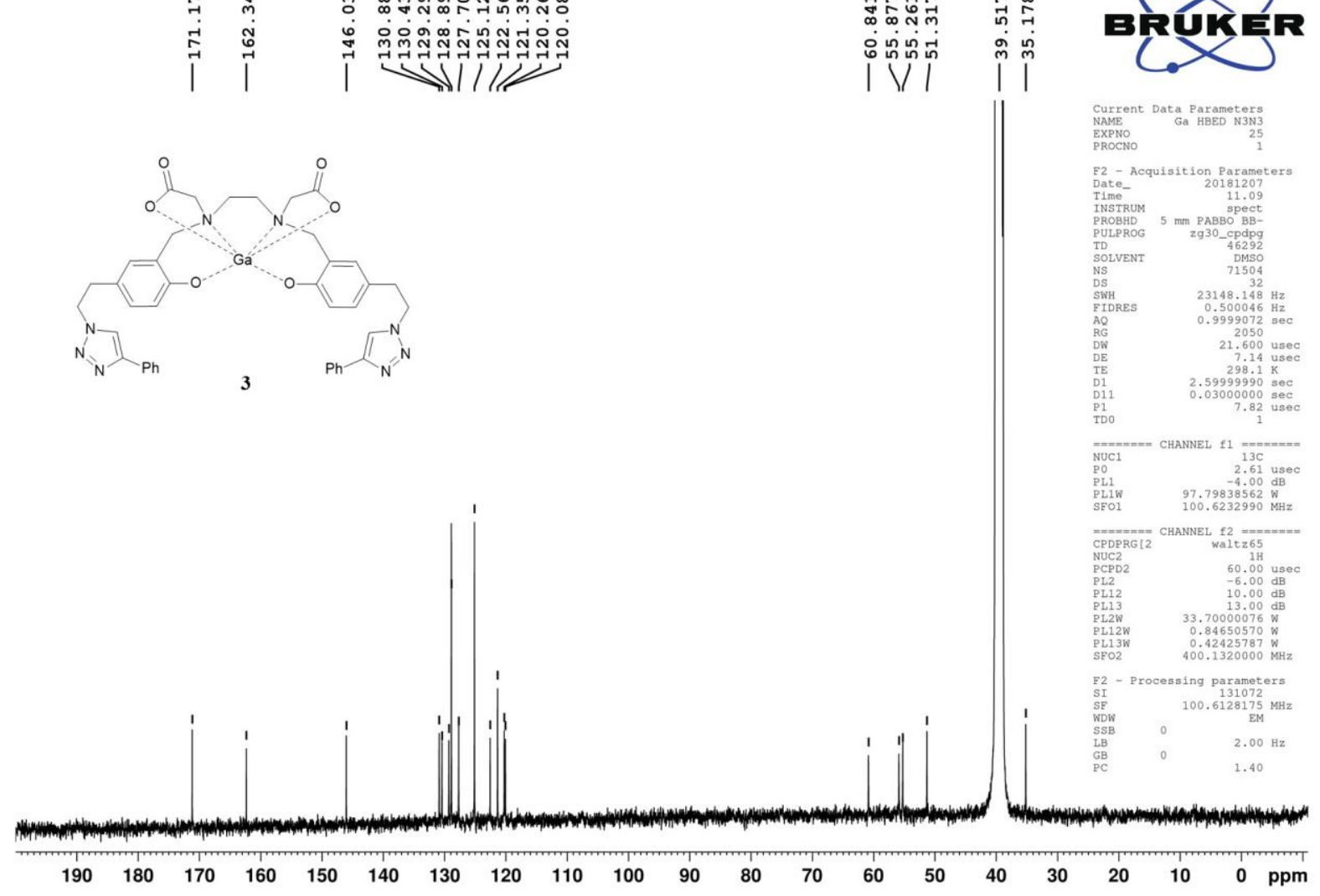

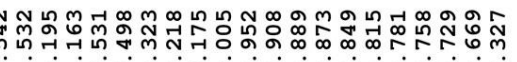

年

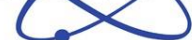

Current Data Parameters
NAME Ga HBED N3N3

EXPNO

F2 - Acquisition Parameter
Date_- 20181207

$\begin{array}{lr}\text { Date_ } & 20181207 \\ \text { Time } & 8.28 \\ \text { INSTUM } & \text { spect }\end{array}$

spect
PROBHD
PULPROG

TD

NS

$\begin{array}{lr}\text { SWH } & 6402.049 \mathrm{~Hz} \\ \text { FIDRES } & 0.070423 \mathrm{~Hz} \\ \text { AQ } & 7.0999150 \mathrm{se}\end{array}$

\begin{tabular}{rr}
$\mathrm{DW}$ & 161 \\
$\mathrm{DE}$ & $18.100 \mathrm{usec}$ \\
$\mathrm{TE}$ & 12.12 usec \\
\hline
\end{tabular}

$1.00000000 \mathrm{sec}$
$9.00 \mathrm{usec}$

$======0$ CHANNEL fI $=======$
NUC1 $1 \mathrm{H}$

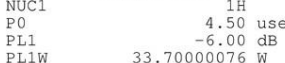

$\begin{array}{ll}33.70000076 \mathrm{~W} \\ \text { SFO1 } & 400.1329400 \mathrm{MHz}\end{array}$

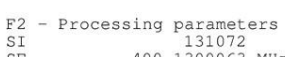

SF $\quad 400.1300063 \mathrm{MH}$

$\begin{array}{lll}\mathrm{SSB} & 0 & 0.30 \mathrm{~Hz} \\ \mathrm{LB} & & \end{array}$

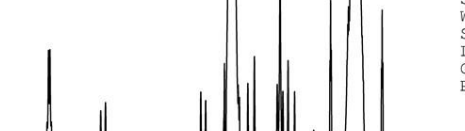

1.00
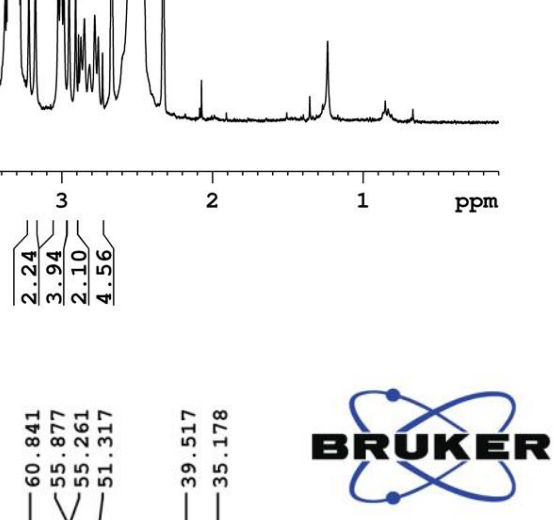
ㅇํํํำ

Current Data Parameters
NAME
EXPNO Ga HBED N3N3
PXOCN

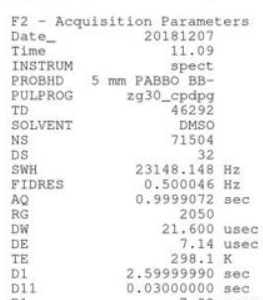

$\underset{\mathrm{P} 1}{\mathrm{PD}}$

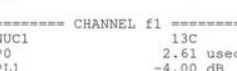

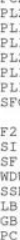


$\operatorname{COSY}\left(\mathrm{d}_{6}\right.$-DMSO)
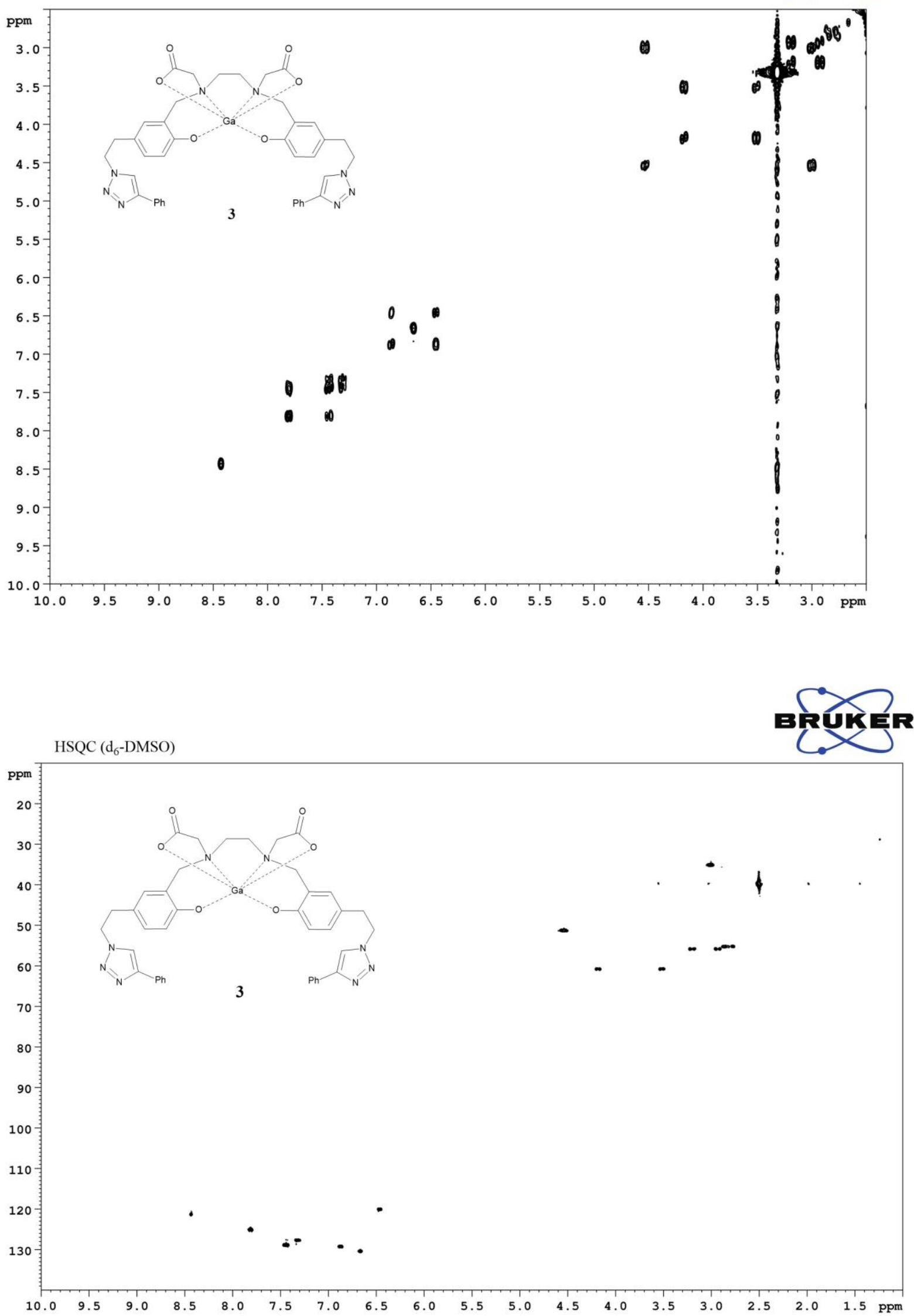

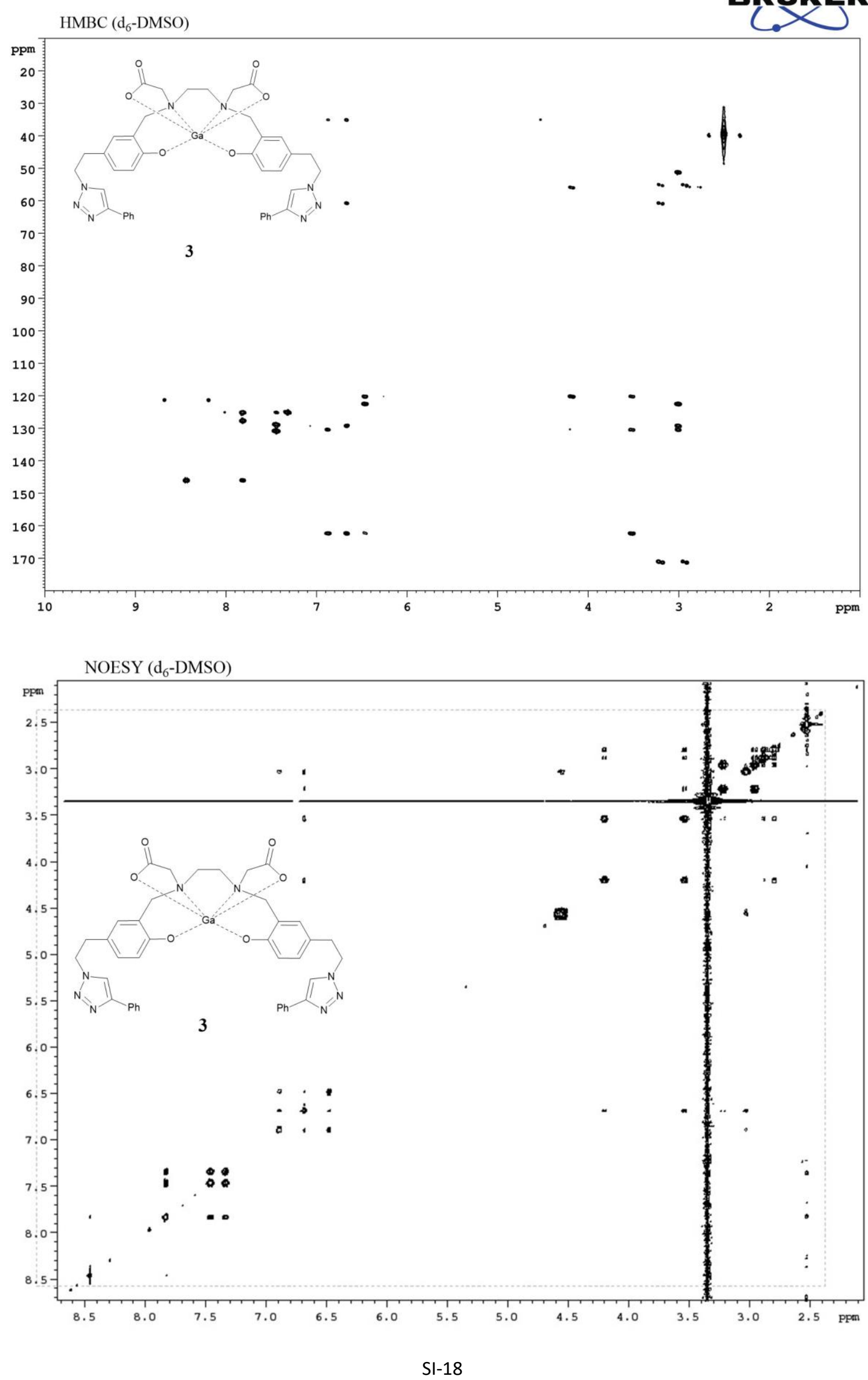